\title{
Gestão do Conhecimento: Um Mapeamento das Principais Publicações Sobre o Assunto para o Levantamento de Temáticas Emergentes
}

Knowledge Management: A Mapping of the Main Publications on the Subject to Identify Emerging Research Lines

\section{Resumo}

objetivo do artigo foi mapear a produção científica internacional sobre a Gestão do Conhecimento na Web of Science no recorte temporal entre os anos 2012 e 2018, identificando os principais artigos e as linhas de pesquisa emergentes sobre o assunto. Trata-se de uma pesquisa do tipo descritiva por meio de um estudo bibliométrico, no qual foram identificados 321 trabalhos publicados nos últimos seis anos no indicador Local Citation Score, que compõem o corpus da análise bibliométrica. Em seguida, os dados coletados foram tratados no software HistCiteTM. Os resultados sinalizaram que: os trabalhos publicados nos últimos seis anos sobre gestão do conhecimento exploram o núcleo intelectual da temática como disciplina acadêmica, para testar se ela apresenta sinais acadêmicos de uma disciplina de referência. As linhas de pesquisa emergentes sinalizam para a necessidade de estudos sobre o impacto da gestão do conhecimento no desempenho econômico organizacional, sobre a influência da gestão do conhecimento na competitividade tecnológica no mercado atual, e sobre examinar o valor prático da gestão do conhecimento.

\section{Abstract}

The objective of the research was to map the international scientific production on Knowledge Management in one of the main databases, Web of Science, in the temporal cut between the years 2012 and 2018 , identifying the main articles and the emerging research lines on the subject in the Local Citation Score. This is a descriptive research through a bibliometric study. 327 papers were published in the last six years in the Local Citation Score that compose the bibliometric analysis corpus were identified. The collected data were then treated in the HistCite ${ }^{T M}$ software. The results indicate that: the work published in the last six years on knowledge management explores the intellectual core of the subject as an academic discipline to test if it represents signs of a reference discipline. Emerging research lines point to the need for studies on the impact of knowledge management on organizational economic performance, on the influence of knowledge management on technological competitiveness in the current market, and on examining the practical value of knowledge management. 


\section{INTRODUÇÃO}

A Gestão do Conhecimento (GC) consiste em processos de conhecimento (criar, compartilhar, adquirir, transferir, aplicar, avaliar e descartar), infraestruturas (TIC), fatores culturais e capacidades de gestão (gestão de pessoas e capacidades dinâmicas) que suportam e melhoram a performance organizacional em busca da inovação e da vantagem competitiva. A GC envolve ainda sistemas operacionais, habilidades locais e transculturais e know-how que são necessários para a resolução de problemas do dia-a-dia e para a manutenção das organizações no futuro (BUKOWITZ; WILLIAMS, 2002; CARDOSO; MEIREILES; PERALTA, 2012; ALEGRE; SENGUPTA; LAPIEDRA, 2013). Como disciplina acadêmica, a GC entrou em pauta apenas em meados dos anos 90. Entretanto, o assunto tem longas raízes históricas, pois têm sido bem documentado na literatura e seus conceitos e aplicações práticas e empíricas remontam há pelo menos 4.000 anos atrás (gregos antigos); enquanto as primeiras tentativas acadêmicas de estabelecer práticas de GC remontam aos estudos de Chaparral Steel no ano de 1975 (SERENKO, 2013).

Apesar de sua evolução precoce, a GC é um constructo de maturação gradual que vêm se tornando uma disciplina de referência com relevantes impactos teóricos assim como práticos (KOENIG; JANK, 2012; SERENKO; BONTIS, 2013b) atraindo a atenção de pesquisadores, profissionais e decisores políticos, com sinais de uma disciplina científica independente (SERENKO; DUMAY, 2015). Destarte, é vital avaliar as tendências de estudo sobre GC investigando as características das publicações sobre o assunto.

Nesse sentido, procurando compreender qual o estado da discussão na academia internacional e as linhas de pesquisa emergentes sobre GC nos artigos mais relevantes, levantou-se a seguinte questão de pesquisa: o que se publica atualmente sobre GC e quais as linhas de pesquisa emergentes sobre o assunto nos manuscritos mais citados na literatura da área?

O objetivo da pesquisa foi mapear a produção científica internacional sobre a Gestão do Conhecimento em uma das principais bases de dados, a Web of Science, no recorte temporal entre os anos 2012 e 2018, identificando os principais artigos e as linhas de pesquisa emergentes sobre o assunto no Local Citation Score.

A escolha da Web of Science (Wos) para a pesquisa se justifica pela abrangência de cobertura em mais de cem áreas do conhecimento científico (MOURA et al., 2017). Por meio da análise bibliométrica foram feitos os seguintes levantamentos: i) top 10 das palavras-chave mais utilizadas nos títulos dos artigos no Local Citation Score (indicador que representa os artigos mais citados apenas na Web of Science); ii) levantamento da distribuição das publicações por ano; iii) os periódicos com mais artigos publicados sobre a temática; iv) os autores com maior número de publicações na temática; v) os países com maior publicação sobre o assunto; vi) top 10 dos artigos mais citados no $L C S$ e; vii) o levantamento das linhas de pesquisa emergentes por meio das sugestões de trabalhos futuros ou das 
limitações apresentadas pelos autores nos estudos.

A pesquisa se justifica por lançar luz sobre novas temáticas em GC evitando-se uma abordagem estreita e reducionista do assunto. Além dessa seção introdutória, a pesquisa apresentou os procedimentos metodológicos utilizados. Na terceira seção foram apresentados os resultados evidenciados, na quarta, debruçou-se sobre a discussão destes achados, e por fim, a quinta seção apresentou as conclusões e limitações com a oportunidade de pesquisas futuras.

\section{PROCEDIMENTOS METODOLÓGICOS}

Os procedimentos metodológicos desta pesquisa foram do tipo exploratório-descritivo por meio de um estudo bibliométrico, uma técnica quantitativa e estatística para medir índices de produção e disseminação do conhecimento. De acordo com Mattar (1996), a pesquisa exploratória dota o pesquisador de maior conhecimento sobre o tema que está sendo abordado ou o problema da pesquisa, sendo usada nos primeiros estágios, quando o investigador busca tomar conhecimento, adquirir familiaridade e compreender melhor os fenômenos e pode ser utilizada como passo inicial para um processo contínuo de estudos.

A importância do estudo bibliométrico é sustentada pela necessidade de se conhecer e avaliar a produtividade, identificar tendências e crescimento, usuários e autores, verificar a cobertura das revistas, medir a disseminação da informação e também formular políticas (SOUZA, 2013), a fim de ampliar a construção do conhecimento para o pesquisador e para a comunidade científica (VALMORBIDA, ENSSLIN; ENSSLIN, 2014).

Portanto, as bibliometrias "servem de cartografia para mapear as origens dos conceitos existentes, apontar as principais lentes teóricas usadas para investigar um assunto e levantar as ferramentas metodológicas utilizadas em trabalhos anteriores" (CHUEKE; AMATUCCI, 2015, p. 1).

Para esta pesquisa, a recuperação dos dados foi feita na base $W o S$ em sua coleção principal. Esta base tem abrangência internacional e contém informações bibliográficas e citações de aproximadamente 40 milhões de artigos científicos, além disso, oferece registros bibliográficos padronizados que possibilita que os dados sejam trabalhados em softwares que auxiliam na contagem, padronização e organização dos dados (SOUZA, 2013).

O período pesquisado foi de seis anos (2012-2018), sendo considerado o intervalo de maior evolução das pesquisas sobre a Gestão do Conhecimento, conforme levantamento feito na WoS. Devido a este crescimento, se faz necessário conhecer as interfaces e os temas emergentes sobre o assunto. O descritor "knowledge management" foi utilizado como sendo o termo que melhor caracteriza o processo de $\mathrm{GC}$, pois "ao perceber o conhecimento enquanto processo, será permitido à empresa gerenciá-lo de maneira a diminuir as incertezas do processo de transferência de conhecimento e integração entre pessoas, entre sistemas ou entre pessoas e sistemas" (FREIRE; SPANHOL, 2014, p. 15). 
A coleta dos dados foi realizada nos títulos dos artigos no período de janeiro e fevereiro de 2019. Utilizou-se ainda os filtros disponíveis na ISI Web of Science, coleção principal para o refinamento da pesquisa na seguinte sequência: i) refinamento por tipo de documento: foi selecionada a opção article; ii) refinamento pelo idioma: foram selecionados os idiomas english, portuguese e french; e, iii) refinamento pelas áreas do conhecimento: foram selecionadas as áreas de management, public administration, business e business finance, pois contemplam conteúdos associados ao descritor base e a área de administração.

Assim, foi possível identificar 321 trabalhos publicados nos últimos seis anos. Utilizou-se o indicador Local Citation Score (LCS) como parâmetro da análise bibliométrica, pois representam citações de autores que abordam a temática da GC citando outros autores que também são referencias no assunto. Portanto, as citações no $L C S$ se referem diretamente ao corpus selecionado na pesquisa bibliométrica. Em seguida, os dados coletados foram tratados no pacote de software de análise bibliométrica HistCite ${ }^{T M}$, sendo realizadas as seguintes análises: levantamento dos dados bibliométricos (artigos publicados, periódicos indexados, autores, instituições de vínculo dos autores, países, referências citadas, citações recebidas pelos artigos), as palavras-chave mais utilizadas nos títulos dos artigos, a distribuição das publicações por ano, os periódicos com mais artigos publicados sobre a temática, os autores com maior número de publicações na temática, os países com mais artigos publicados na temática, o top 10 dos artigos mais citados na $W o S$, os as sugestões de trabalhos futuros sinalizadas nos principais periódicos sobre a temática.

Além destes dados, foram elucidados aspectos dos textos dos dez artigos mais citados localmente, no intuito de identificar suas principais contribuições para a temática de Gestão do Conhecimento, pois os estudos bibliométricos não estão mais somente centrados na métrica, mas também na contextualização da produção científica (TEIXEIRA et al., 2013). Os resultados das análises e a discussão dos resultados estão apresentados em seguida.

\section{RESULTADOS E DISCUSSÃO DOS RESULTADOS}

Os 321 artigos localizados sobre GC estão publicados em 77 periódicos indexados na base acadêmica e foram escritos por 730 autores (somando-se autores principais e co-autores) que possuem vínculos com 417 instituições de ensino localizadas em 58 países. Os artigos publicados receberam 210 citações no corpus selecionado para esta pesquisa. Na Tabela 1, a seguir, são apresentados esses resultados. 
Tabela 1 - Resultado do levantamento bibliométrico

\begin{tabular}{lc}
\hline \multicolumn{1}{c}{ Dados bibliométricos } & Quantidades \\
\hline Publicações (artigos) & 321 \\
\hline Periódicos indexados & 77 \\
\hline Autores & 730 \\
\hline Instituições (vínculos dos autores) & 417 \\
\hline Países & 58 \\
\hline Citações recebidas pelos artigos $(L C S)$ & 319 \\
\hline
\end{tabular}

Fonte: Elaborado pelos autores a partir de dados da Web of Science (2018).

A evolução anual das publicações sobre GC apresentou uma média aproximada de 53 manuscritos publicados por ano, no período de 2012-2018. O periódico internacional mais representativo para a temática é o Journal of Knowledge Management com 122 (38\%) dos registros de publicações, que possui classificação de H-index Scopus 63, conforme a tabela 2, tendo o fator de impacto de 2,551 no Journal Citation Reports e A1 no Qualis CAPES Brasil, representando, assim um periódico de elevada qualidade e impacto.

Tabela 2 - Top 10 dos periódicos com mais artigos publicados sobre a temática

\begin{tabular}{lccc}
\hline \multicolumn{1}{c}{ Periódicos } & $\begin{array}{c}\text { H-index } \\
\text { Scopus }\end{array}$ & $\begin{array}{c}\text { Quantidade } \\
\text { de Artigos }\end{array}$ & $\%$ \\
\hline $\begin{array}{l}\text { Journal of Knowledge Management } \\
\text { Knowledge Management Research \& }\end{array}$ & 63 & 122 & 38,0 \\
Practice & 24 & 52 & 16,1 \\
\hline $\begin{array}{l}\text { Journal of Business Research } \\
\text { International Journal of Project }\end{array}$ & 133 & 10 & 3,1 \\
Management & 103 & 8 & 2,5 \\
Management Decision & 57 & 6 & 1,8 \\
\hline Information \& Management & 128 & 5 & 1,5 \\
Business Process Management & 50 & 5 & 1,5 \\
Journal & 46 & 4 & 1,2 \\
\hline Journal of Intellectual Capital & 48 & 4 & 1,2 \\
\hline Service Industries Journal & 17 & 3 & 0,9 \\
\hline Baltic Journal of Management & & & \\
\hline
\end{tabular}

Fonte: Elaborado pelos autores a partir de dados da Web of Science (2018).

Após análise dos periódicos, foram identificados os autores que possuem a maior quantidade de registros de publicações sobre GC. Nesse sentido se destacam: Aino Kianto, com nove publicações, e Alexander Serenko com oito.

Dentro do corpus selecionado, tem-se a relação entre os artigos mais citados, indicando, dentre 
estes, quais apresentam conexão de citações (linhas que conectam os círculos) e também quais são os mais citados no grupo (tamanhos diferentes dos círculos), conforme Figura 1.

Figura 1 - Top 10 dos artigos mais citados no grupo de artigos selecionados (LCS) na faixa temporal pesquisada.

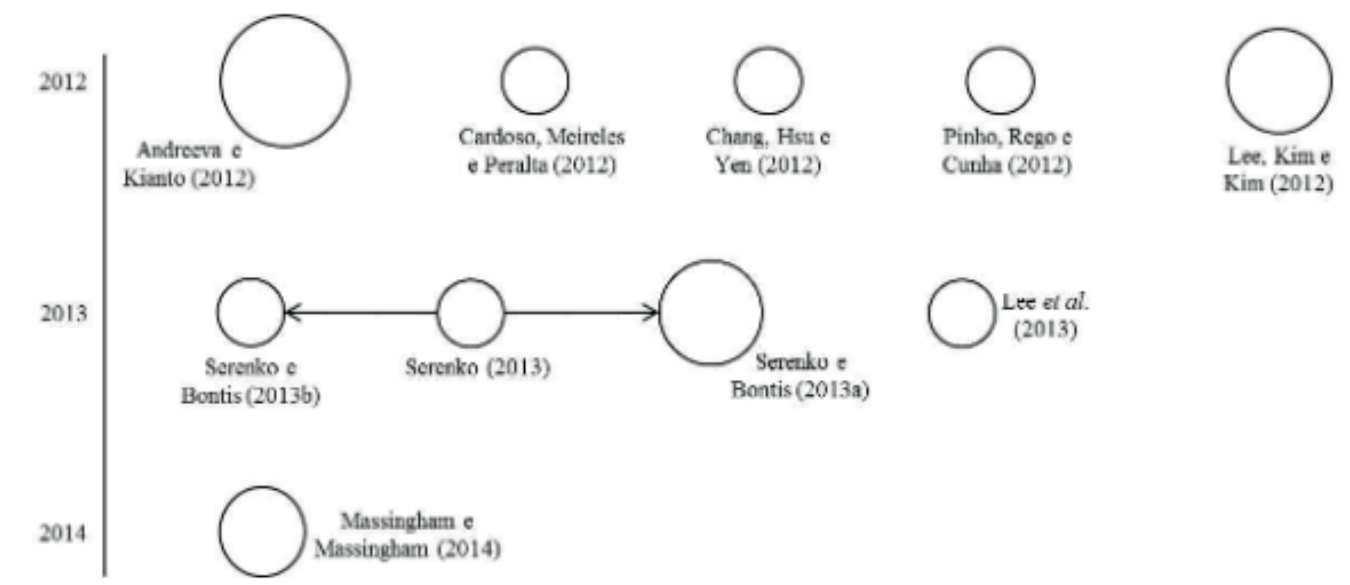

Fonte: Elaborado pelos autores a partir de dados da Web of Science (2018).

Nesse sentido, pode-se observar que, na análise da Figura 1, complementada pela Tabela 3, o trabalho de Andreeva e Kianto (2012) é o mais citado. Outro destaque é que a pesquisa de Serenko (2013) aparece como um artigo de ligação entre os trabalhos de Serenko e Bontis (2013a) e Serenko e Bontis (2013b), sendo do próprio autor em parceria.

Tabela 3 - Top 10 dos trabalhos mais citados no conjunto de artigos selecionados nesta pesquisa $(L C S)$

\begin{tabular}{|c|c|c|c|}
\hline $\begin{array}{c}\text { Citações } \\
\text { locais }\end{array}$ & Títulos dos Trabalhos & Fonte das Publicações & Referências \\
\hline 21 & $\begin{array}{l}\text { Does knowledge management really matter? } \\
\text { Linking knowledge management practices, } \\
\text { competitiveness and economic performance }\end{array}$ & $\begin{array}{c}\text { Journal of Knowledge } \\
\text { Management, } 16(4): 617-636\end{array}$ & $\begin{array}{c}\text { Andreeva e } \\
\text { Kianto (2012) }\end{array}$ \\
\hline 12 & $\begin{array}{l}\text { An integrated view of knowledge } \\
\text { management for performance }\end{array}$ & $\begin{array}{l}\text { Journal of Knowledge } \\
\text { Management, } 16 \text { (2): 183-203 }\end{array}$ & $\begin{array}{l}\text { Lee, Kim e } \\
\text { Kim (2012) }\end{array}$ \\
\hline 11 & $\begin{array}{l}\text { Global ranking of knowledge management } \\
\text { and intellectual capital academic journals: } \\
2013 \text { update }\end{array}$ & $\begin{array}{l}\text { Journal of Knowledge } \\
\text { Management, } 17 \text { (2): } 307-326\end{array}$ & $\begin{array}{c}\text { Serenko e } \\
\text { Bontis (2013a) }\end{array}$ \\
\hline 7 & $\begin{array}{c}\text { Does knowledge management produce } \\
\text { practical outcomes? }\end{array}$ & $\begin{array}{c}\text { Journal of Knowledge } \\
\text { Management, } 18 \text { (2): } 221-254\end{array}$ & $\begin{array}{l}\text { Massingham e } \\
\text { Massingham } \\
\text { (2014) }\end{array}$ \\
\hline 6 & $\begin{array}{l}\text { The intellectual core and impact of the } \\
\text { knowledge management academic discipline }\end{array}$ & $\begin{array}{l}\text { Journal of Knowledge } \\
\text { Management, } 17 \text { (1): 137-155 }\end{array}$ & $\begin{array}{c}\text { Serenko e } \\
\text { Bontis (2013b) }\end{array}$ \\
\hline 6 & $\begin{array}{l}\text { Meta-analysis of scientometric research of } \\
\text { knowledge management: discovering the } \\
\text { identity of the discipline }\end{array}$ & $\begin{array}{c}\text { Journal of Knowledge } \\
\text { Management, } 17 \text { (5): } 773-812\end{array}$ & Serenko (2013) \\
\hline 5 & $\begin{array}{l}\text { Improving knowledge management } \\
\text { processes: a hybrid positive approach }\end{array}$ & $\begin{array}{c}\text { Journal of Knowledge } \\
\text { Management, } 16(2): 215-242\end{array}$ & $\begin{array}{l}\text { Pinho, Rego e } \\
\text { Cunha (2012) }\end{array}$ \\
\hline 5 & $\begin{array}{l}\text { Knowledge management and its critical } \\
\text { factors in social economy organizations }\end{array}$ & $\begin{array}{c}\text { Journal of Knowledge } \\
\text { Management, } 16(2): 267-284\end{array}$ & $\begin{array}{c}\text { Cardoso, } \\
\text { Meireles e } \\
\text { Peralta (2012) }\end{array}$ \\
\hline 5 & $\begin{array}{l}\text { Factors affecting knowledge management } \\
\text { success: the fit perspective }\end{array}$ & $\begin{array}{l}\text { Journal of Knowledge } \\
\text { Management, } 16(6): 847-861\end{array}$ & $\begin{array}{l}\text { Chang, Hsu e } \\
\text { Yen (2012) }\end{array}$ \\
\hline 5 & $\begin{array}{l}\text { Knowledge management: a key determinant } \\
\text { in advancing technological innovation? }\end{array}$ & $\begin{array}{c}\text { Journal of Knowledge } \\
\text { Management, } 17 \text { (6): 848-872 }\end{array}$ & $\begin{array}{l}\text { Lee, Leong, } \\
\text { Hew e Ooi } \\
(2013)\end{array}$ \\
\hline
\end{tabular}

Fonte: Elaborado pelos autores a partir de dados da Web of Science (2018). 
O trabalho de Andreeva e Kianto (2012) foi o mais citado, no qual os autores buscaram analisar, por meio de uma pesquisa em 234 empresas da Finlândia, Rússia e China (países emergentes), a relação entre as práticas de GC, competitividade empresarial e o desempenho econômico e financeiro. Andreeva e Kianto (2012) descobriram que as práticas de gestão de pessoas (práticas sociais) e as TICs (práticas técnicas) tem correlação estatística forte e influenciam os resultados financeiros e a competitividade das empresas. Descobriam ainda que as práticas de TIC promovem um melhor desempenho financeiro para as organizações apenas quando estão associadas com as práticas sociais; e que a gestão do conhecimento realmente funciona como um facilitador de alto desempenho organizacional.

O trabalho de Lee, Kim e Kim (2012) foi o segundo mais citado. Os autores buscaram analisar a relação entre infraestruturas de GC (fatores técnicos [tecnologias], estruturais e culturais [mecanismos normativos, de confiança e de facilitação de aprendizagem] que permitem a maximização do capital social), processos de conhecimento (aquisição, conversão, aplicação, aprendizagem e proteção do conhecimento), aprendizagem e desempenho organizacional. Como resultados foi percebido que : a infraestrutura de GC afeta os processos de conhecimento e os processos de conhecimento mediam a relação entre a infraestrutura de GC e o desempenho organizacional, o que demonstra a relevância da infraestrutura de GC para o desempenho organizacional.

Já o manuscrito de Serenko e Bontis (2013a) teve como objetivo atualizar um ranking global de GC e de Capital Intelectual (GC/CI) de periódicos acadêmicos. Os autores classificaram 25 periódicos para a temática $(\mathrm{GC} / \mathrm{CI})$, dos quais, seis foram os mais importantes: Journal of Knowledge Management, Journal of Intellectual Capital, The Learning Organization, Knowledge Management Research \& Practice, Knowledge and Process Management e International Journal of Knowledge Management, semelhante aos resultados encontrados nesta pesquisa.

A Gestão do Conhecimento produz resultados práticos? É com esse título que Massingham e Massingham (2014) apresentam seu trabalho. Os autores iniciaram o estudoabordando os benefícios que a GC pode fornecer às organizações, relacionando-os ao retorno sobre o investimento (tempo necessário para recuperar o investimento em GC), à melhoria da governança corporativa, à melhoria do processo de negócio (eficiência e eficácia), entre outros, e terminam apresentando sete resultados práticos da GC que são propostos como métodos para persuadir os gerentes a investir na GC, são eles: a curva de aprendizagem (descreve que os aumentos de produção são resultantes da aprendizagem organizacional), experiência da curva (busca explicar que a rentabilidade do negócio é derivada da experiência adquirida pela organização no mercado), alinhamento estratégico (refere-se aos sistemas de controle de gestão eficazes que permitem a implementação da estratégia, tais como: Activity Based Costing e o Balanced Scorecard), conectividade (refere-se aos ganhos de produtividade gerados pelo capital social), gestão de riscos (qualidade dos resultados da GC), gestão de valor (se a GC melhora a percepção dos stakeholders sobre o valor da organização) e o contrato psicológico (se a GC melhora 
a moral e a produtividade dos trabalhadores).

O artigo de Serenko e Bontis (2013b) buscou, em duplo objetivo, explorar o núcleo intelectual da GC como disciplina acadêmica para testar se ela representa sinais de uma disciplina de referência, e analisar o impacto teórico e prático da disciplina. Os autores descobriram que a disciplina de GC constrói o seu conhecimento principalmente em relatórios de pesquisa publicados no idioma inglês; que usa praticamente apenas livros e revistas no desenvolvimento da teoria de GC; que converte conhecimento experimental em conhecimento acadêmico; mas ainda não é uma disciplina de referência, no entanto, está em fase de consolidação e não é uma moda científica, corroborando com a visão apresentada por Serenko e Bontis (2013a). Entretanto, segundo estes autores "mais pesquisas são necessárias para entender melhor o desenvolvimento do estado e futuro da disciplina de GC" (p. 142).

Serenko (2013), desta vez em autoria solo, realizou uma meta-análise de pesquisas cienciométricas sobre o campo da GC, analisando 108 estudos cienciométricos. Segundo o autor, o volume de estudos tem crescido ao passo de até dez publicações por ano, entretanto suas descobertas às vezes são inconsistentes, pois existem obras que são publicadas em revistas que não estão centradas em GC ou muitas vezes os pesquisadores estão isolados. Segundo Serenko (2013, p. 773) "a disciplina de GC tem raízes históricas profundas. Ela sofre de um elevado grau de diferentes interpretações e é representada por fluxos de pesquisas diferentes". De modo geral, o autor afirma que evitando as inconsistências e focando nos principais periódicos de referência sobre o assuntoenquanto disciplina, a temática exibe atributos de um domínio acadêmico saudável sem anomalias aparentes e está progredindo em direção à maturidade acadêmica.

Com o objetivo de identificar e discutir as barreiras e facilitadores tecnológicos, sócio-organizacionais e individuais para quatro processos de GC (aquisição, criação, compartilhamento e transferência), Pinho, Rego e Cunha (2012) realizaram uma revisão da literatura na WoS, EBSCO, Emerald e ProQuest e descobriram queos fatores que afetam (barreiras ou facilitadores) os processos de GC envolvem de alguma forma o capital social e a interação entre tecnologias e as necessidades e atividades dos usuários. "Além da tecnologia e das variáveis de capital social, outros fatores como liderança, cultura orientada para o desempenho, formação e práticas de desenvolvimento e habilidades T-shaped emergiram como relevantes para os processos de GC" (PINHO; REGO; CUNHA, 2012, p. 216). Por fim, os autores colocaram que a GC prospera em contextos culturais favoráveis para a aquisição, criação, compartilhamento e transferência do conhecimento, quando existem tecnologias e sistemas de suporte ao armazenamento, compartilhamento, direcionamento do conhecimento para as pessoas certas; existe uma boa comunicação e coordenação interna e externa; existe uma liderança positiva (que fomenta o compromisso e o alto desempenho); quando existe educação, treinamento e práticas de desenvolvimento humano (habilidades de pesquisa, trabalho em equipe, entre outras); e, quando existe bom relacionamento e o desenvolvimento de habilidade T-shaped (conhecimento de várias disciplinas, capacidade de escrever, falar outras línguas, de comunicação e de publicar conhecimento). 
Cardoso, Meireiles e Peralta (2012), procuraram apresentar e validar empiricamente um modelo conceitual para organizações da economia solidária para interpretar o papel (facilitador ou inibidor) do comprometimento organizacional, da cultura centrada no conhecimento e das práticas formativas na GC. O estudo foi desenvolvido junto a organizações portuguesas pertencentes ao setor da economia solidária. Os resultados sinalizaram queorganizações de economia solidária que desenvolvempráticas formativas de GC devem estar alinhadas com o comprometimento organizacional e devem basear suas políticas de formação em uma cultura centrada no conhecimento. Além disso, os autores descobriram que o comprometimento afetivo possui um efeito positivo na cultura centrada no conhecimento (efeito facilitador) e consequentemente sobre as práticas de GC formais e informais; já o comprometimento instrumental mostrou-se um inibidor à GC. Por conseguinte, identificaram que a cultura centrada no conhecimento tem correlação positiva com as práticas formativas para a GC (tanto formais, quanto informais), pois segundo os autores "se uma organização é capaz de criar uma cultura centrada no conhecimento, terá criado a condição mais facilitadora possível para o sucesso das práticas formais e informais de GC" (CARDOSO; MEIREILES; PERALTA, 2012, p. 277).

Já o trabalho de Chang, Hsu e Yen (2012), teve como objetivo desenvolver quatro perfis teóricos ideais dos processos de GC (socialização, externalização, combinação e internalização) e das capacidades do sistema de gerenciamento do conhecimento (SGC) (capacidade de codificação e de rede) para subunidades organizacionais com base nas características de suas tarefas, focadas e orientadas para processos, focadas e orientadas para conteúdos, tarefas amplas e orientadas para processos e tarefas amplas e orientadas para conteúdos. O estudo empírico de método quali-quanti foi desenvolvido em 12 subunidades de uma empresa aeroespacial intensiva em conhecimento e engenharia. Os achados sinalizaram que os ajustes entre os processos, as capacidades do sistema de gerenciamento do conhecimento e as características das tarefas (se focada ou ampla) podem melhorar o desempenho da GC. Segundo os autores, isto sugere que:

[...] não é suficiente para a alta administração de uma organização simplesmente investir para implementar sistemas de informação para a GC. Em vez disso, é necessário compreender a natureza das características das tarefas das subunidades antes que o SGC seja implantado. (CHANG; HSU; YEN, 2012, p. 856).

Por fim, Lee et al. (2013) se propuseram a analisar a relação entre as práticas de GC e sua relação com as inovações tecnológicas no setor de manufatura da Malásia. Por meio do método quantitativo os autores perceberam que as práticas de GC de compartilhamento, aplicação e armazenamento do conhecimento tem relação positiva e significativa com a inovação tecnológica (de produto e inovação de processo), assim como, as inter-relações entre as práticas de GC são positivas e significantes. A prática de aquisição do conhecimento não demonstrou significância, portanto, a aquisição do conhecimento não estimula a inovação tecnológica.

No contexto dos manuscritos mais citados no $L C S$, conforme a Tabela 4, são linhas de pesquisa 
emergentes, levantadas por meio das sugestões trabalhos futuros ou das limitações apresentadas nos estudos: estudar a interface entre constructos do comportamento humanos e organizacional, tais como: confiança (LEE; KIM; KIM, 2012), liderança (PINHO; REGO; CUNHA, 2012), comprometimento (CARDOSO; MEIREILES; PERALTA, 2012), cultura (CARDOSO; MEIREILES; PERALTA, 2012; PINHO; REGO; CUNHA, 2012), entre outros, com a GC por meio da construção de modelos de mensuração; explorar o núcleo intelectual da GC como disciplina acadêmica para testar se ela apresenta sinais de uma disciplina de referência (SERENKO; BONTIS, 2013B), e analisar o impacto teórico e prático da disciplina na literatura (MASSINGHAM; MASSINGHAM, 2014); e estudar o impacto da GC sobre a competitividade e o desempenho econômico-financeiro das organizações (ANDREEVA; KIANTO, 2012; LEE; KIM; KIM, 2012; CHANG; HSU; YEN, 2012).

\section{CONCLUSÕES}

Após a análise dos dados encontrados nos principais artigos internacionais, os resultados respondem ao problema de pesquisa identificando publicações, predominantemente associadas aos processos táticos da GC, assim como linhas de pesquisa emergentes sobre o assunto. Os trabalhos publicados nos últimos seis anos no $L C S$ sobre GC exploram o núcleo intelectual da GC como disciplina acadêmica para testar se ela representa sinais de uma matéria de referência e se exibe atributos de um domínio acadêmico saudável sem anomalias aparentes, e buscam medir o valor da GC na prática.

As linhas de pesquisa ou lacunas emergentes sugeridas nos principais manuscritos para o desenvolvimento de futuras pesquisas, e que poderão contribuir para a consolidação da GC como uma disciplina de referência, ainda repousam, em sua maioria, sob o paradigma funcionalista, em torno do impacto da GC sobre o desempenho econômico organizacional, sobre a sua influência na competitividade do mercado atual; e sobre desenvolver estudos para examinar o seu valor prático.

Pelos principais estudos analisados e pelas descobertas levantadas, as pesquisas empíricas rigorosas e longitudinais e as meta-análises são sugestões metodológicas reiteradas de alguns dos autores estudados, juntamente à métodos estruturados de triangulação de dados objetivando validar as proposições teóricas, para garantir a utilidade, o impacto e a sustentabilidade da GC como uma disciplina acadêmica.

Por fim, a exclusividade de uso da base de dados ISI Web of Science pode ser apresentada nestas conclusões tanto como uma limitação de alcance, quanto como um indicativo de possibilidades de ampliação deste estudo. Como sugestões para pesquisas futuras, além das já pontuadas como objetivo desse trabalho sugere-se a realização de pesquisas em outras bases de dados, a exemplo da Scopus, pois um estudo comparativo poderá identificar possíveis alterações de percurso teórico ou metodológico dos investigadores e das suas formas de abordar este campo ou objeto de estudo, já que se trata de uma disciplina em amadurecimento e construção. 


\section{Agradecimentos}

O presente trabalho foi realizado com apoio da Coordenação de Aperfeiçoamento de Pessoal de Nível Superior - Brasil (CAPES) - Código de financiamento 001.

\section{Referências}

ALEGRE, J.; SENGUPTA, K.; LAPIEDRA, R.. Knowledge management and innovation performance in a high-tech SMEs industry. International Small Business Journal, v. 31, n. 4, p. 454-470, 2013.

ANDREEVA, T.; KIANTO, A. Does knowledge management really matter? Linking knowledge management practices, competitiveness and economic performance. Journal of Knowledge Management, v. 16, n. 4, p. 617-636, 2012.

BUKOWITZ, W. R.; WILLIAMS, R. L. Manual de gestão do conhecimento: ferramentas e técnicas que criam valor para a empresa. Bookman, 2002.

CARDOSO, L.; MEIRELES, A.; PERALTA, C. F. Knowledge management and its critical factors in social economy organizations. Journal of Knowledge Management, v. 16, n. 2, p. 267-284, 2012. CASTRO, A. B. C. de; BRITO, L. M. P.; VARELA, J. H. de S. A ressignificação da área de gestão de pessoas e os novos papéis das pessoas e das organizações. HOLOS, v. 33, n. 4, p. 408-423, 2017. CHANG, C.-M.; HSU, M.-H.; YEN, C.-H. Factors affecting knowledge management success: the fit perspective. Journal of Knowledge Management, v. 16, n. 6, p. 847-861, 2012.

CHUEKE, G. V.; AMATUCCI, M. O que é bibliometria? uma introdução ao fórum. Internext, v. 10, n. 2, p. 1-5, 2015.

FREIRE, P. de S.; SPANHOL, F. J. O conhecimento organizacional: Produto ou processo?. Perspectivas em Gestão \& Conhecimento, v. 4, n. 1, p. 3-21, 2014.

KOENIG, M. E. D.; JANK, D. A. The (common) sense of KM. Journal of Information \& Knowledge Management, v. 11, n. 02, p. 1250009, 2012.

LEE, S.; KIM, B. G.; KIM, H. An integrated view of knowledge management for performance. Journal of Knowledge Management, v. 16, n. 2, p. 183-203, 2012.

LEE, V. H., LEONG, L. Y., HEW, T. S., OOI, K. B. Knowledge management: a key determinant in advancing technological innovation?. Journal of Knowledge Management, v. 17, n. 6, p. 848-872, 2013.

MASSINGHAM, P. R.; MASSINGHAM, R. K. Does knowledge management produce practical outcomes?. Journal of Knowledge Management, v. 18, n. 2, p. 221-254, 2014.

MOURA, L. K. B., de MESQUITA, R. F., Mobin, M., MATOS, F. T. C., Monte, T. L., Lago, E. C., ... e SOUSA, L. R. M. Uses of Bibliometric Techniques in Public Health Research. Iranian Journal of 
Public Health, v. 46, n. 10, p. 1435-1436, 2017.

PINHO, I.; REGO, A.; CUNHA, M. P. Improving knowledge management processes: a hybrid positive approach. Journal of Knowledge Management, v. 16, n. 2, p. 215-242, 2012.

SERENKO, A.; DUMAY, J. Citation classics published in knowledge management journals. Part I: articles and their characteristics. Journal of Knowledge Management, v. 19, n. 2, p. 401-431, 2015.

; BONTIS, N. Global ranking of knowledge management and intellectual capital academic journals: 2013 update. Journal of Knowledge Management, v. 17, n. 2, p. 307-326, $2013 \mathrm{a}$.

; . The intellectual core and impact of the knowledge management academic discipline. Journal of Knowledge Management, v. 17, n. 1, p. 137-155, 2013 b.

. Meta-analysis of scientometric research of knowledge management: discovering the identity of the discipline. Journal of Knowledge Management, v. 17, n. 5, p. 773-812, 2013.

SOUZA, Cláudia D. de. A organização do conhecimento: Estudo bibliométrico na base de dados ISI Web of Knowledge. Biblios, n. 51, 2013.

VALMORBIDA, S. M. I., ENSSLIN, S. R., ENSSLIN, L., RIPOLL-FELIU, V. M. Avaliação de desempenho para auxílio na gestão de universidades públicas: análise da literatura para identificação de oportunidades de pesquisas. Contabilidade, Gestão e Governança, v. 17, n. 3, 2014.

WANG, S.; NOE, R. A.; WANG, Z. Motivating knowledge sharing in knowledge management systems: A quasi-field experiment. Journal of Management, v. 40, n. 4, p. 978-1009, 2014. 